\title{
Detección de infección por VIH a través de test rápido en mujeres embarazadas: Una estrategia exitosa para disminuir su transmisión vertical
}

\author{
JORGE QUIAN R., ANA VISCONTI G., STELLA GUTIÉRREZ R., ANA GALLI R., MARÍA MATURO R., \\ VIRGINIA GALEANO R., MARGARITA SERRA T. y MARTA LIONI H.
}

\section{Detection of HIV infection in pregnant women by rapid testing: a successful strategy to reduce its vertical transmission}

A high percentage of Uruguayan pregnant women are not under medical control. As a consequence, vertically transmission of HIV infection reaches to $50 \%$. The aim of this study was to know the prevalence of HIV infection in pregnant women who did not know their serological status and to decrease mother-to-child transmission. Patients and Methods: from January 2002 to January 2004 the HIV rapid test was performed to every pregnant woman that assisted to a public Uruguayan hospital unaware of her condition. The proper prophylactic decisions were adopted according to gestational age. The newborn infants were classified according to CDC criteria. HIV infection prevalence in pregnant women and in their newborn infants was calculated. Results: there were 34.338 obstetric consultations and 4.599 rapid tests were performed. Fifty-nine turned out positive in 58 women, 8 of them knew their serological status previously. The HIV infection prevalence was 1.1\% (IC95\% 0.8-1.4). Five cases were discharged: 1 false positive and 3 miscarriages and 1 abortion. Ten women dropped out in the follow up. Twelve women received TARV during pregnancy for over a week. Thirty-nine infants could be controlled: 33 seroreverted, 4 were exposed and 2 became infected. Mother-to-child transmission was 5.1\%. If all patients who dropped out the follow up were infected, the transmission rate should be of $20.4 \%$; therefore the infection would have been prevented in 16 children. As many women and children were lost, other complementary actions as counseling and social worker interview should be adopted in order to improve the yield of rapid test screening strategy.

Key words: HIV; rapid test; mother-to-child transmission.

Palabras clave: VIH, test rápido, transmisión vertical.

\section{Introducción}

El objetivo principal de los equipos de salud es evitar la enfermedad. Las campañas de vacunación en la población pediátrica y general son el más claro ejemplo de estas políticas ${ }^{1}$. Contra la infección por el virus de la inmunodeficiencia humana (VIH), aún no han podido lograrse avances significativos en el diseño de una vacuna, pese a los esfuerzos que se realizan ${ }^{2}$.

\footnotetext{
Universidad de la República:

Departamento de Pediatría (JQR, SGR).

Clínica Obstétrico-Ginecológica (AVG).

Centro Hospitalario Pereira Rossell (AGR, MLH).

Laboratorios Abbott (MMR).

Programa Nacional de SIDA Ministerio de Salud Pública (MST, VGR).
}

Recibido: 13 de mayo 2005

Aceptado: 30 agosto 2005

Proyecto financiado por el Programa de las Naciones Unidas para el Desarrollo (PNUD) y el Programa Nacional de SIDA dependiente del Ministerio de Salud Pública. 
En el ámbito pediátrico la infección por VIH de adquisición vertical es virtualmente evitable si se logran algunos objetivos básicos: el control profesional de la mujer embarazada y la realización de la quimioprofilaxis con fármacos antiretrovirales (ARV) en la madre gestante infecta$\mathrm{da}^{3-6}$.

En Uruguay, el 30\% de los nacimientos asistidos en el Centro Hospitalario Pereira Rossell (CHPR) provienen de embarazos mal controlados. Su causa es multifactorial siendo las más determinantes una falta de accesibilidad a los centros de salud, ignorancia y marginación social. Por otra parte, el $99 \%$ de los nacimientos son institucionales y el CHPR es la principal maternidad del área de Salud Pública del Uruguay. Uno de cada 6 uruguayos nace en él ${ }^{7}$.

En esta población, las cifras de transmisión vertical del VIH en mujeres que no controlan su embarazo alcanzan al 50\% ${ }^{8}$. Estas mujeres e hijos en gestación no reciben los beneficios de la profilaxis: no son tratadas con ARV de acuerdo a su condición de infección por VIH, no tienen la posibilidad de una cesárea programada, sus hijos no reciben AZT de acuerdo al protocolo de Connor $\mathrm{y} \mathrm{col}^{3}$ y además son amamantados por la madre prolongando el riesgo de infectarse más allá del parto.

Cuando las mujeres no controlan su embarazo desde el inicio y no acceden a la detección del VIH por los métodos convencionales, se pueden planificar estrategias alternativas como la realización de un test rápido para detectar la infección por $\mathrm{VIH}$, si ella ignora su estado serológico ${ }^{9-10}$. La realización de este test se considera una estrategia adecuada para disminuir la transmisión vertical ya, que de acuerdo a la edad gestacional en que se encuentre la mujer, se pueden aplicar distintas medidas de profilaxis ${ }^{11}$.

Los objetivos del presente estudio fueron:

- Detectar la prevalencia de VIH en la población de mujeres embarazadas sin control o mal controladas que consultaron al Servicio de Emergencia Obstétrico-Ginecológico del CHPR.

- Disminuir la transmisión vertical tomando las medidas necesarias de acuerdo a la edad gestacional en que se diagnosticó la infección materna por VIH.

\section{Pacientes y Métodos}

En el período comprendido entre el 10 de enero de 2002 y el 9 de enero de 2004 se realizó en el Servicio de Emergencia Obstétrico-
Ginecológico del CHPR un test rápido para detectar anticuerpos anti VIH a toda mujer embarazada consultante en dicho servicio, cualquiera fuera su edad gestacional, y que ignorara su estado serológico.

El test se realizó previo un consentimiento oral de la paciente (se consideró la mejor estrategia pues muchas mujeres llegaron en etapas avanzadas del trabajo de parto).

En caso de ser positivo, las conductas adoptadas dependían de la situación obstétrica.

Si la embarazada concurría durante la gestación, se la conectaba con la policlínica obstétrico-pediátrica de seguimiento para infección por $\mathrm{VIH}$ que funciona en el CHPR. Una vez captada se le ofrecían quimioprofilaxis con ARV y se discutía la necesidad de una cesárea programada. Si el diagnóstico se realizaba durante el trabajo de parto, el obstetra indicaba zidovudina (AZT) intravenoso durante el mismo, fuera vaginal o cesárea; en algunos pacientes se utilizó además nevirapina vía oral. En ambos casos, el recién nacido recibía AZT y se evitaba el amamantamiento materno ${ }^{3}$.

Se observaron todas las pautas del protocolo nacional de atención neonatal para la prevención de la transmisión vertical de VIH, elaboradas por el Ministerio de Salud Pública ${ }^{12}$.

Si el parto acaecía fuera del hospital (parto en ciudad, pudiendo ser en el domicilio o en centros periféricos no preparados para asistencia profesional de partos) y la madre concurría en el puerperio inmediato, se prescribía AZT al recién nacido y se evitaba la lactancia materna.

Los hijos de las mujeres infectadas por VIH continuaban su control en la policlínica de seguimiento de niños infectados por VIH del CHPR.

Realización del test. Fue llevado a cabo por estudiantes medicina que cursan su último año curricular, los que recibieron previamente instrucción teórico-práctica por un médico laboratorista (M.M.R.) para la realización del test. Una vez efectuado el test, era completado un registro con el nombre de la paciente, número de su documento de identidad, fecha de realización, resultado y firma del técnico responsable.

Descripción del test rápido. Se utilizó Determine HIV 1/2® (Abbott Laboratories, Abbott Park, Illinois USA), prueba inmunocromatográfica para la detección cualitativa de anticuerpos antiVIH-1 y VIH-2 con sensibilidad y especificidad cercanas a $100 \%$, de acuerdo a indicaciones del fabricante. En breve, se procedió a aplicar $50 \mu \mathrm{l}$ de sangre obtenida por punción digital sobre la tira reactiva. La presencia de anticuerpos antiVIH se evidencia por la formación de una línea 
roja en la "ventana" del paciente. En cada test se incluye una "ventana de control", donde se forma una línea roja validante del resultado. La lectura se efectúa a los 15 minutos en forma visual. Las alternativas de resultado son: positivo, negativo o inválido. Los resultados positivos fueron confirmados mediante técnicas de ELISA y western blot o inmunofluorescencia en el Laboratorio Nacional de Referencia.

Clasificación de los niños. Los hijos de madre infectadas por VIH fueron categorizados en: infectado (I), con seroreversión (S) o con exposición perinatal (E), de acuerdo a criterios establecidos por el CDC de Atlanta, E.U.A. ${ }^{13}$.

Análisis estadístico. Se determinó la prevalencia de casos diagnosticados en esa población con su respectivo intervalo de confianza y el porcentaje de transmisión vertical.

\section{Resultados}

Durante el período de estudio hubo 34.338 consultas obstétricas; se realizó test rápido a 4.599 mujeres $(13,4 \%$ del total) que desconocían su estado serológico. Nacieron 18.286 niños.

Detección de infección por VIH en mujeres embarazadas. Se detectó 59 resultados positivos $(1,28 \%)$ en 58 mujeres (una mujer tuvo dos embarazos). Cinco mujeres conocían su condición serológica pero la negaron y a 3 se les realizó el test por no tener documentación que confirmara esta condición. La prevalencia de infección por VIH en mujeres que desconocía su serología en el embarazo fue de 1,1\% (IC 95\% $0,8-1,4)$. De los 59 resultados positivos, se detectó un falso positivo que también tuvo un ELISA convencional falso positivo; se descartó por western blot. El niño no fue incorporado al seguimiento y la madre no se incluyó dentro del análisis.

Se detectaron 6 resultados inválidos.

La edad de las mujeres fue en promedio de 30 años (rango: 16 a 46 años).

Tabla 1. Edad gestacional al momento del diagnóstico de infección por $\mathrm{VIH}$

\begin{tabular}{lr}
\hline$\cdot 1^{\circ}$ trimestre: & 4 \\
$\bullet 2^{\circ}$ trimestre: & 7 \\
$\cdot 3^{\text {o }}$ trimestre: & 34 \\
$\cdot$ Puerperio inmediato: & 3 \\
- Dato no consignado: & 10 \\
Total & 58 \\
\hline
\end{tabular}

El diagnóstico de infección por VIH se hizo en el primer trimestre del embarazo en 4 casos, en el segundo trimestre en $7 \mathrm{y}$ en el tercer trimestre se diagnosticó en 34 (16 antes de las 38 semanas y 18 entre las semanas 38 y 42). En el puerperio inmediato se detectó la infección en 3 pacientes. No quedó registrado el dato en 10 casos (Tabla 1).

Treinta y un mujeres (54\%) habían concurrido una vez a control obstétrico pero desconocían su condición respecto a la infección por VIH. Cuarenta y cuatro mujeres $(77 \%)$ tenían más de una gestación previa, 4 eran primigestas y en 10 casos no quedó registrado el dato.

Seguimiento del embarazo. Cuatro mujeres abortaron (3 abortos espontáneos y uno provocado) y fueron derivadas al Programa Nacional de SIDA para asesoramiento de su enfermedad. De los restantes 54 embarazos, 10 mujeres se perdieron en el seguimiento desconociéndose la evolución posterior. Se intentó localizar a estas mujeres que no concurrieron a los controles correspondientes pero los datos del domicilio, así como de los documentos de identidad registrados cuando se realizó el test, fueron falsos.

Terapia anti retroviral durante la gestación. Doce mujeres recibieron ARV durante el embarazo por más de una semana (zidovudina en 12, AZT y lamivudina en 2 y una recibió triple terapia con zidovudina, nelfinavir y lamivudina). Cuatro mujeres iniciaron el tratamiento ARV en el segundo trimestre y ocho en el tercero.

En 32 casos no se pudo prescribir ARV por vía oral por ingresar al servicio en período expulsivo o puerperio. No quedó registrado el dato en 10 casos (Tabla 2).

Vía de resolución del parto. La resolución del parto en las 44 mujeres que continuaron su seguimiento fue vía vaginal en 18 casos (16 en el CHPR, 1 en domicilio y 1 en otra institución de salud), cesárea de urgencia en 14 y cesárea programada en 12 casos (Tabla 3 ).

Tabla 2. Uso de TARV en 58 mujeres embarazadas e infectadas por VIH

\begin{tabular}{ll} 
- Usaron TARV durante el embarazo & 12 \\
$\quad\left(4\right.$ en $2^{\circ}$ trimestre y 8 en el $\left.3^{\circ}\right)$ & \\
- No usaron TARV durante el embarazo & $32^{*}$ \\
- No se registró el dato & 10 \\
Total & $54^{* *}$ \\
\hline TARV: terapia antiretroviral \\
* No recibieron TARV porque consultaron en parto o \\
puerperio inmediato \\
* De los 58 embarazos estudiados 4 mujeres abortaron
\end{tabular}


Tabla 3. Vía de resolución del parto de las 54 mujeres infectadas por VIH

- Vaginal: 18

(16 en hospital, 1 en domicilio,

1 en otro centro)

- Cesárea de urgencia:

- Cesárea programada:

- Se perdieron del seguimiento:
Tabla 4. Uso de TARV durante el parto

- Zidovudina $\mathrm{i} / \mathrm{v}$

(de 30 minutos a 12 horas)

- Zidovudina + nevirapina oral

- No recibieron

- No se registró el dato

- Se perdieron del seguimiento

Total 54
Parto y terapia antiretroviral. Durante el parto vaginal o cesárea 31 mujeres recibieron AZT intravenoso con un mínimo de 30 minutos a un máximo de 12 horas; en 14 casos fue asociado a nevirapina vía oral. Ocho no recibieron ARV y de 15 se desconoce el dato por no constar en la historia (5) o porque fueron perdidas en el seguimiento (10) (Tabla 4).

\section{Transmisión vertical de la infección}

Estimación teórica: el total de niños que debió haber sido controlado fue de 54 si todos hubieran nacido; considerando que el porcentaje de transmisión en nuestro país es de alrededor de $50 \%$, se habría producido infección vertical en 27 niños. Frecuencia de transmisión observada: No hubo nacimientos gemelares. De los 44 recién nacidos se controlaron 39 niños, de término en 35 casos y 4 menores de 37 semanas. Todos recibieron AZT vía oral y no fueron amamantados. En la evolución, 33 sero-revirtieron, 2 se confirmaron como infectados y 4 permanecen como expuestos porque abandonaron los controles (Figura 1). De estos 4 expuestos, uno que tenía dos RPC-VIH negativas, tuvo una muerte súbita en su domicilio y no se obtuvo necropsia; 2 niños tenían dos RPC-VIH negativas al momento de abandonar controles y 1 tenía una sola RPC-VIH negativa anterior al cuarto mes de vida. Solamente hubo 2 infectados en los 39 controlados (porcentaje de transmisión de 5,1\%).

Uno de los dos niños infectados provenía de una madre de 16 años, primigesta, que tuvo su primer contacto con el equipo de salud en un

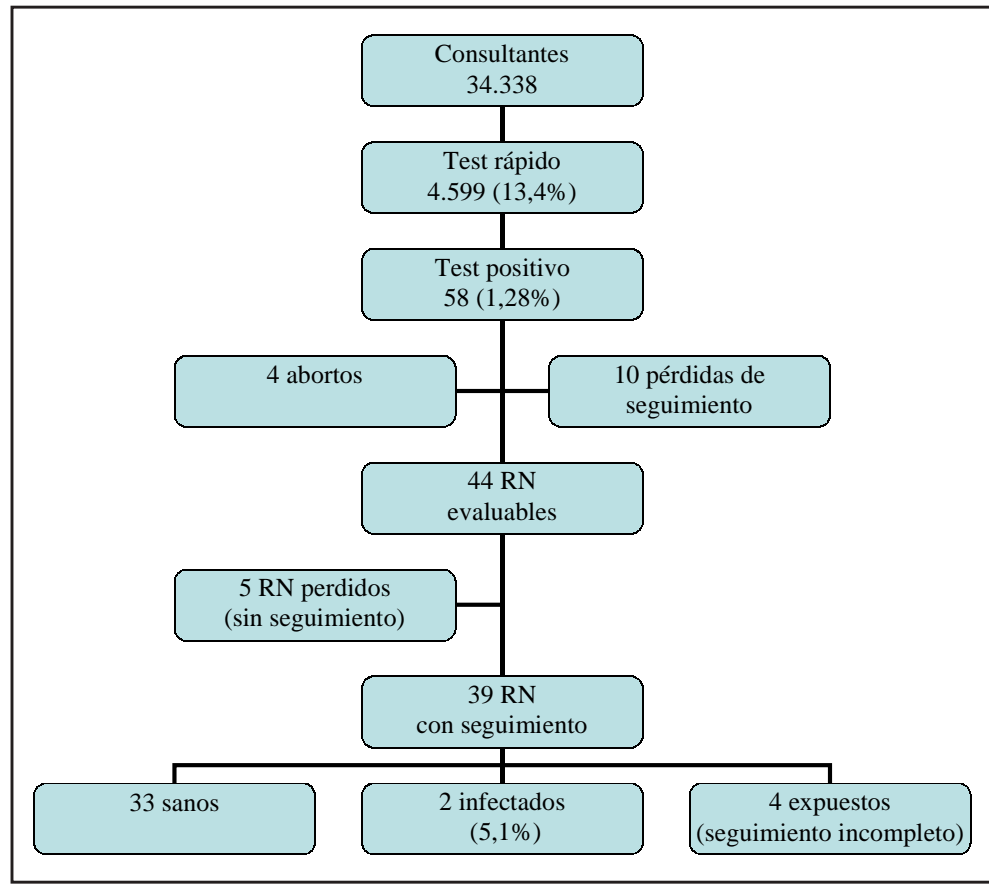

Figura 1. Transmisión vertical de la infección por VIH. 
centro de primer nivel de atención a las 20 semanas de gestación y no supo su condición de infección por VIH hasta haberse realizado el test rápido cuando ingresó en trabajo de parto. El nacimiento fue a las 40 semanas por cesárea de urgencia. Recibió nevirapina v/o AZT endovenoso durante 7 horas. La recién nacida pesó $3.360 \mathrm{~g}$, recibió $\mathrm{AZT}$ v/o durante 42 días y no fue amamantada. El otro infectado era un recién nacido de $2.375 \mathrm{~g}$; llegó en el puerperio inmediato de un parto realizado en un centro periférico. Recibió AZT v/o y no fue amamantado.

Si sumamos 2 probables infectados entre los 4 expuestos más 7 probables infectados de los 15 que nunca concurrieron, el número total de infectados hubiera sido 11. En ese caso el porcentaje de transmisión en 54 recién nacidos hipotéticos hubiera sido del 20,4\% y, en forma teórica, se evitó la infección de 16 niños.

\section{Discusión}

En Uruguay, la prevalencia de infección por VIH en la población general entre los años 2002 y 2004 fue de $0,36 \%{ }^{14}$. La prevalencia en las mujeres embarazadas que consultaron por diferentes causas en el Servicio de Emergencia ObstétricoGinecológico, y que no conocían u ocultaron su estado serológico para VIH $(13,4 \%$ del total consultante) fue más alta que en la población general: 58 mujeres positivas $(1,28 \%)$.

El medio del cual provienen, con muy poco acceso a la educación, con severos problemas económicos y marginados de los sistemas de salud, puede haber sido determinante en esta prevalencia. Por otra parte, las mujeres que no controlan su embarazo o lo hacen en forma inadecuada, que son portadoras de infección por VIH no estudiada ni tratada y que muchas veces tienen además enfermedades secundarias a adicción a drogas, podrían tener cifras de abortos superiores a la población general, calculándose que en Uruguay 4 de cada 10 embarazos terminan en un aborto provocado ${ }^{15}$.

El número de mujeres y niños perdidos durante el seguimiento de este estudio es significativamente alto. Muchas mujeres dieron domicilios y documentación falsa (se les preguntaba el número de documento de identidad pero no se exigía su presentación) por lo que fue imposible identificarlas ni localizarlas posteriormente. Fue un error por parte del equipo de salud que diseñó el estudio pensar que la instrumentación del test rápido por sí sola, al mejorar el diagnóstico de infección, mejoraría el cuidado en salud de todos los pacientes. El informe del resultado del test rápido lo hizo el personal que estaba de guardia; ninguno había recibido instrucciones sobre consejería para actuar en estos $\operatorname{casos}^{16}$. La consejería es un pilar fundamental para asistir a un paciente infectado por el VIH, y será necesario incluirlo en el futuro.

El temor a la discriminación sigue existiendo en estas pacientes. Esa podría ser la razón por la cual mujeres que conocían su condición de infectadas, lo negaron al concurrir a la emergencia.

Treinta y un mujeres habían contactado previamente un equipo de salud para control del embarazo y sin embargo, desconocían su condición serológica. En Uruguay desde 1997 existe un decreto ley que obliga a ofrecer la realización del test de ELISA para VIH. Se concluye que la sola existencia del decreto ley no alcanza para cierta población. Se debería analizar las causas por las cuales el personal de salud no indicó los controles correspondientes o, en el caso de haberlos solicitado, se debe investigar la causa de su no realización.

La transmisión vertical en el grupo de 39 niños con seguimiento fue de 5\%; esta disminución de la transmisión vertical a partir del test rápido estimula y compromete a seguir realizando acciones en el futuro. La estimación teórica realizada a partir de cifras históricas en esta población ${ }^{8}$ evitó la infección en 16 niños por lo que se logró el cumplimiento del objetivo planteado: disminuir la transmisión vertical de las mujeres embarazadas que no controlan o controlan mal su embarazo.

De acuerdo a lo establecido en la literatura médica, la mayor parte de la transmisión materno-fetal ocurre en el período perinatal y hasta $15 \%$ de la transmisión se produce durante la lactancia materna ${ }^{17}$. La aplicación del test rápido detectando serología positiva en el último trimestre del embarazo y en el periparto, permite tomar medidas que impactan fuertemente en la transmisión vertical ${ }^{5}$.

Entre las medidas adoptadas para disminuir esta transmisión, a la prescripción de ARV y la suspensión del amamantamiento, se debe agregar la inhibición farmacológica de la lactancia en la madre y la administración de leches de vaca modificadas durante los primeros meses de vida.

\section{Conclusiones}

La aplicación de la estrategia del test rápido detectó un 1,1\% de mujeres embarazadas infectadas por VIH que desconocían su condición. La 
transmisión vertical de la infección fue de $5 \%$. Con esta estrategia se evitó, en una estimación teórica, la infección de 16 niños. La pérdida de seguimiento de mujeres embarazadas y sus hijos, probablemente disminuya aún más si la realización del test rápido es complementada con una necesaria consejería en el momento del diagnóstico, hecha por un profesional entrenado.

\section{Resumen}

En Uruguay existe un gran porcentaje de mujeres que no controlan su embarazo y donde la transmisión vertical de la infección por VIH alcanza al $50 \%$. Objetivo: conocer la prevalencia de infección por VIH en mujeres embarazadas que desconocen su estado serológico, y que no controlan o controlan mal su gestación, y disminuir la transmisión vertical. Pacientes y métodos: entre enero de 2002 y enero de 2004 se realizó el test rápido para detectar infección por VIH a toda mujer embarazada que no supiera su condición serológica y que consultara en el Servicio Gineco-Obstétrico del Centro Hospitalario Pereira Rossell. De acuerdo a la edad gestacional se tomaban las medidas profilácticas consideradas más adecuadas. Se calculó la prevalencia de infección por VIH en esas mujeres y el porcentaje de transmisión a sus hijos. Resultados: hubo 34.338 consultas obstétricas y se realizaron 4.599 tests rápidos. Se detectaron 58 mujeres positivas, 8 de ellas conocían previamente su condición serológica. La prevalencia de infección por VIH fue de 1,1\% (IC95\% 0,8-1,4). Hubo un falso positivo y cuatro abortos. Diez mujeres abandonaron los controles. Doce recibieron antiretrovirales durante el embarazo por más de una semana. Se controlaron 39 niños y 2 fueron infectados. El porcentaje de transmisión fue de $5,1 \%$. Como algunas mujeres y niños se perdieron en el seguimiento, es necesario complementar la estrategia de un screening de test rápido para infección por VIH con otras medidas como consejería y entrevista con asistente social para optimizar estos resultados.

\section{Referencias}

1.- Plotkin S L, Plotkin S A. Chapter 1. A short history of vaccination. En Plotkin SA, Orenstein WA. Vaccines $4^{\text {th }}$ edition. Philadelphia: Elsevier INC. USA. 2004; 115.

2.- Popovic V, Gurwith M, Francis D, Harro C, Mayer K H. Differential efficacy in prevention of HIV infection by $\operatorname{rgp} 120 \mathrm{HIV}-1$ vaccine by gender and race: is it real? $43^{\text {rd }}$ Interscience Conference on Antimicrobial Agents and Chemotherapy. ICAAC Abstracts H-1942.
2003 Sep. Chicago, USA 144-17.

3.- Connor E, Sperling R, Gelber R, Kiselev P, Scott G, O'Sullivan M, et al. Reduction of maternal-infant transmission of HIV type-1 with zidovudine treatment. N Engl J Med 1994: 331: 1173-80.

4.- Aleixo L.Goodenow M. Sleasman J. Zidovudine administered to women infected with HIV type 1 and to their neonates reduces pediatric infection independent of an effect on levels of maternal virus. $\mathrm{J}$ Pediatr 1997; 130: 906-14.

5.- Wade N, Birkhead G,Warren B, Charbonneau T, French T, Wang L, et al. Abbreviated regimens of zidovudine prophylaxis and perinatal transmission of the HIV. N Engl J Med 1998; 339 (20): 1409-14.

6.- McGowan J. Crane M. Wiznia A. Blum S. Combination antiretroviral therapy in HIV-infected pregnant women. Obstet Gyenecol 1999; 94: 641-6.

7.- El Centro Hospitalario Pereira Rossell en Cifras. Año 2002. Sistema de Información Hospitalario. Dirección Hospital Pediátrico.dirhpedi@adinet.com.uy (accedido 30 noviembre 2003)

8.- Quian J, Gutiérrez S, Picon T, Visconti A, González A, Nin M, et al. Transmisión madre-hijo del VIH según quimioprofilaxis recibida, vía de nacimiento y amamantamiento. Rev Med Uruguay 2002; 18: 265-9.

9.- Irwin K, Olivo N, Scable C,Weber J, Janssen R, Ernst J, et al. Performance characteristics of a rapid HIV antibody assay in a hospital with a high prevalence of HIV infection. Ann Intern Med 1996; 125: 471-5.

10.- Downing R, Otten R, Marum E, Biryahwaho B, Alwano-Edyegu M, Sempala S, et al. Optimizing the delivery of HIV counseling and testing services: the Uganda experience using rapid HIV antibody test algorithms. J Acquir Immune Defic Syndr 1998; 18 (4): 384-8.

11.- Public Health Service Task Force. Recommendations for use of antiretrovirals drugs in pregnant HIV-1infected women for maternal health and interventions to reduce perinatal HIV-1 transmission in the United States. Nov 26, 2003. http://AIDSinfo.nih.gov (accedido 14 noviembre 2004)

12.- Ministerio de Salud Pública. Organización Panamericana de la Salud. Programa de las Naciones Unidas Sobre el SIDA. Terapia Antiretroviral para adultos y niños. Recomendaciones del comité de expertos en VIH-SIDA. Uruguay 1996.

13.- 1994 Revised classification system for HIV infection in children less than 13 years of age. Morb Mortal Wkly Rep MMWR, 1994; 43 No.RR-12:1-13.

14.- Programa Nacional de SIDA. Ministerio de Salud Pública. República Oriental del Uruguay. Informe epidemiológico. Mayo/2004.

15.- Briozzo L. Aborto provocado: un problema humano. Perspectivas para su análisis. Estrategias para su reducción. Rev Med Uruguay 2003; 19: 188-200.

16.- Malonza I, Richardson B, Kreiss J, Bwayo J, Stewart G. The effect of rapid HIV-1testing on uptake of perinatal HIV-1 interventions: a randomized clinical trial. AIDS 2003; 17 (1): 113-8.

17.- Nduati R, John G, Mbori-Ngacha D, Richardson B, Overbaugh J, Mwatha A, et al. Effect of breastfeeding and formula feeding on transmission of HIV-1: a randomized clinical trial. JAMA 2000; 283: 1167-74.

Correspondencia a:

Jorge Quian Rivero

jorgeq@internet.com.uy 